\title{
Influence of material inhomogeneity and non-linear mechanical behavior of the material on delamination in multilayered beams
}

\author{
Victor Rizov \\ Department of Technical Mechanics, University of Architecture, Civil Engineering and Geodesy, 1 Chr. Smirnensky blvd., 1046 \\ - Sofia, Bulgaria \\ V_RIZOV_FHE@UACG.BG
}

\begin{abstract}
The delamination fracture in four-point bending beams made of adhesively bonded lengthwise vertical layers is studied assuming that each layer exhibits smooth material inhomogeneity along the width and length of the layer. The study aims at determining the strain energy release rate with applying the Ramberg-Osgood equation for modeling the non-linear mechanical behavior of the material in each layer. Cosine laws are used to describe the continuous variation of the modulus of elasticity in width and length directions of layers. Beams made of an arbitrary number of vertical layers which have individual widths and material properties are considered. Besides, the delamination crack is located arbitrary between layers, i.e. the two crack arms have different widths. The J-integral is applied for verification of the non-linear solution to the strain energy release rate derived in the present paper. The solution is used to investigate the influence of material inhomogeneity in width and length directions of layers, the crack location along the beam width, the non-linear mechanical behavior of the material and the crack length on the delamination fracture behavior. The approach developed is expected to be useful in structural design of multilayered inhomogeneous beams with considering the delamination fracture behavior.
\end{abstract}

KEYWORDS. Multilayered beam; Material inhomogeneity; Delamination fracture; Non-linear mechanical behavior of the material.

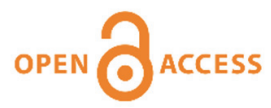

Citation: Rizov, V., Influence of material inhomogeneity and non-linear mechanical behavior of the material on delamination in multilayered beams, Frattura ed Integrità Strutturale, 47 (2019) 468-481.

Received: 31.10 .2018

Accepted: 17.12 .2018

Published: 01.01.2019

Copyright: (C) 2017 This is an open access article under the terms of the CC-BY 4.0, which permits unrestricted use, distribution, and reproduction in any medium, provided the original author and source are credited.

\section{INTRODUCTION}

S tructural members and components made of adhesively bonded layers of dissimilar materials are extensively used in various applications in aerospace and civil engineering mainly when high performance is required [1-3].

Various studies of adhesively bonded joints of fiber reinforced composites and sandwich structures have been reviewed in [1]. The influence of different factors such as joint configuration, adhesive properties, preparation of surfaces and environment factors on the joint behavior has been evaluated and discussed thoroughly. Methods and approaches for predicting the failure behavior have been presented too. The application of fracture mechanics for analyzing of adhesively 
bonded joints has also been considered. It has been shown the importance of using of reliable failure criteria for widening the application of adhesively bonded joints in load-bearing composite and sandwich structural members and components in different industries [1].

A through review of recent publications on failure behaviour of different type of fiber reinforced composite materials has been presented in [2]. Various aspects of fatigue and life prediction of fiber reinforced composites have been discussed in detail. The effects of inherent and environmental factors such as temperature, moisture and corrosion on fatigue and failure of composites have been analyzed. A summary of commonly used failure criteria for life prediction of composite structures subjected to static and fatigue load conditions has been reported. A progressive fatigue model has also been considered in the review. Models for prediction of residual mechanical properties of composites under different loading conditions have been described and discussed [2].

The thermomechanical behaviour of a carbon fiber composite laminated sheet that is irradiated by using a continuous wave chemical oxygen iodine laser has been analyzed in [3]. A potential delamination of the composite sheet makes the analysis of the influence of the laser an important practical task. The temperature distribution in the composite has been studied. The peak temperature of irradiated region has been evaluated. It has been found that the model developed is suitable for simulating of laser ablation of carbon fiber epoxy composite materials. The study has indicated that the laser beam machining is an appropriate process for manufacturing of fiber reinforced composite materials [3].

However, multilayered materials and structures have low interlaminar strength which is a premise for development of delamination cracks $[4,5]$. Delamination fracture or separation of layers is the predominant failure mode of multilayered structures.

The service lifetime of laminated composite structural members and components is limited by their delamination fracture behavior under certain loading conditions [4]. Delamination fracture of multilayered material systems under mode II crack loading conditions with considering of the creep behavior has been studied in [4]. For this purpose, the methods of linear elastic fracture mechanics have been applied. An elevated temperature has been used to accelerate the delamination fracture under constant external loads. Delamination behavior of a double cantilever beam configuration has been analyzed. By using of the Paris power law, a methodology for predicting the service lifetime of multilayered beam structures in terms of service load, temperature and initial delamination crack length has been developed [4].

A review of techniques for modeling and analysis of functionally graded single layers and sandwich beam configurations has been presented in [5]. Various solutions which are based on the assumption for linear-elastic behavior of the functionally graded material have been presented and discussed. Analyses of beam structures under both static and dynamic loading conditions have been considered. Free and forced vibrations of functionally graded sandwich constructions have been studied. Investigations of functionally graded sandwich beams resting on two-parameter elastic foundation have been reported. Studies of buckling behaviour of sandwich beams have been reviewed too. Various analyses of bending behaviour of viscoelastic sandwich structures have been discussed. Works dealing with static analyses of functionally graded sandwich beams resting on a Pasternak elastic foundation have also been presented. Solutions of linear-elastic beams made of functionally graded materials under tension and bending have been considered [5].

The main goal of the present paper is to derive the strain energy release rate for a delamination crack in multilayered fourpoint bending beam configurations assuming that each layer exhibits smooth material inhomogeneity in width and length directions. The beam under consideration is made of an arbitrary number of adhesively bonded lengthwise vertical layers which have non-linear mechanical behavior of the material that is treated by applying the Ramberg-Osgood equation. It should be mentioned that in his previous works, the author has studied delamination fracture behavior of various multilayered beam structures made by lengthwise vertical inhomogeneous layers usually by applying power law stress-stain relations for modeling the non-linear mechanical behavior of the material $[6,7]$.

The solution to the strain energy release rate derived in the present paper can be applied in fracture mechanics based structural design of multilayered beams made of inhomogeneous materials such as functionally graded materials which have been widely used in recent years as advanced structural materials in many engineering applications $[8,9,10,11,12,13,14$, $15]$.

\section{DETERMINATION OF THE STRAIN ENERGY RELEASE RATE}

A multilayered four-point bending beam configuration containing a delamination crack of length, $2 a$, is shown schematically in Fig. 1.

The external loading consists of two vertical forces, $F$, applied at the two ends of the beam. The beam crosssection is a rectangle of width, $b$, and height, $h$. The length of the beam is $2\left(l_{1}+l_{2}\right)$. It is assumed that the beam is made 
of adhesively bonded lengthwise vertical layers which exhibit material inhomogeneity in both width and length directions. The number of layers is arbitrary. Each layer has individual width and material properties. Besides, each layer exhibits nonlinear mechanical behavior of the material which is treated by applying the Ramberg-Osgood equation. A notch of depth, $b_{2}$, is introduced in the right-hand lateral surface of the beam in order to generate conditions for delamination fracture. The delamination crack is located symmetrically with respect to the mid-span. Besides, the delamination crack is located arbitrary between vertical layers. Therefore, the cross-sections of the two crack arms have different widths denoted by $b_{1}$ and $b_{2}$ for the left-hand and right-hand crack arms, respectively. The notch divides the right-hand crack arm in two symmetric segments of length, $a$, each. Apparently, the two segments of the right-hand crack arm are free of stresses. It should also be mentioned that the delamination crack is located in the beam portion, $B_{2} B_{4}$, which is loaded in pure bending (Fig. 1).

Due to the symmetry, only half of the beam, $l_{1}+l_{2} \leq x_{3} \leq 2\left(l_{1}+l_{2}\right)$, is considered in the present fracture analysis.

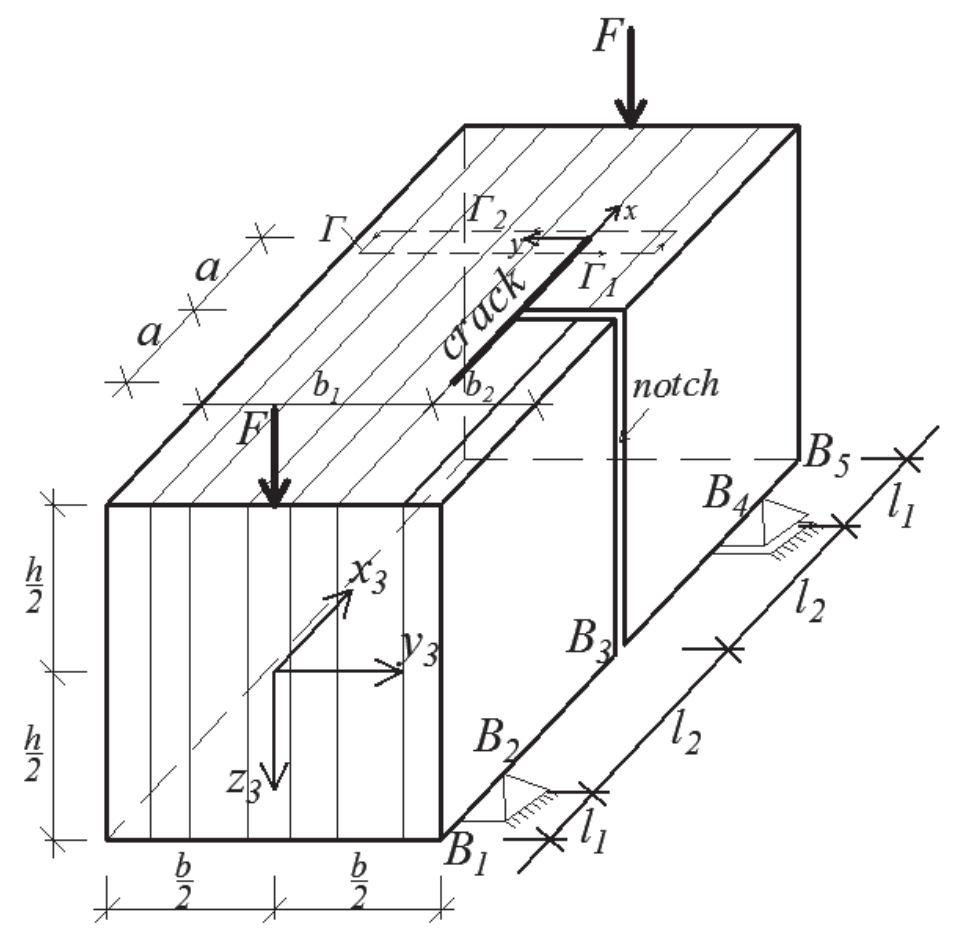

Figure 1: Loading and geometry of the multilayered four-point bending beam configuration.

The delamination fracture behavior is studied in terms of the strain energy release rate, $G$. For this purpose, the strain energy release rate is written as [16]

$$
G=\frac{d U^{*}}{d A}
$$

where $d U^{*}$ is an elementary change of the complementary strain energy, $d A$ is an elementary increase of the delamination crack area. Since

$$
d A=b d a
$$

formula (1) takes the form

$$
G=\frac{d U^{*}}{b d a}
$$


where $d a$ is an elementary increase of the delamination crack length.

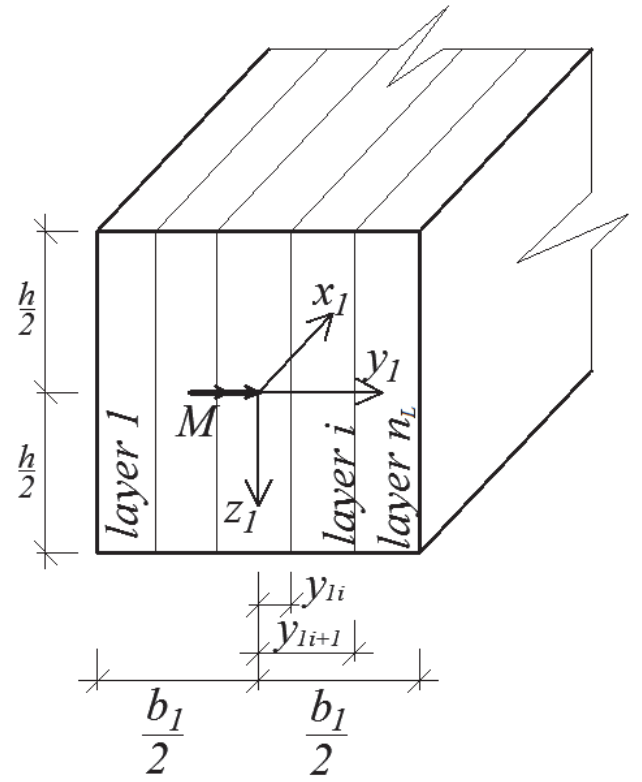

Figure 2: Cross-section of the left-hand crack arm in the beam mid-span.

Since the delamination crack is located in beam portion, $B_{2} B_{4}$, the complementary strain energy cumulated in the beam portions, $B_{1} B_{2}$ and $B_{4} B_{5}$, does not depend on the delamination crack length (Fig. 1). Thus, it is enough to calculate the complementary strain energy cumulated in beam portion, $B_{3} B_{4}$, only. Since the two segments of the right-hand crack arm are free of stresses, the complementary strain energy, $U^{*}$, is written as

$$
U^{*}=U_{L}^{*}+U_{R}^{*}
$$

where $U_{L}^{*}$ and $U_{R}^{*}$ are the complementary strain energies cumulated in the left-hand crack arm and the un-cracked beam portion, $l_{1}+l_{2}+a \leq x_{3} \leq l_{1}+2 l_{2}$.

The complementary strain energy cumulated in the left-hand crack arm is expressed as

$$
U_{L}^{*}=\sum_{i=1}^{i=n_{L}} \int_{0}^{a} \int_{y_{1 i}}^{y_{1 i+1}} \int_{-\frac{h}{2}}^{\frac{h}{2}} u_{0 L_{i}}^{*} d x_{1} d y_{1} d z_{1}
$$

where $n_{L}$ is the number of layers in the left-hand crack arm, $y_{1 i}$ and $y_{1 i+1}$ are the coordinates, respectively, of the lefthand and right-hand lateral surfaces of the $i$-th layer, $u_{0 L_{i}}^{*}$ is complementary strain energy density in the same layer, the axes, $x_{1}, y_{1}$ and $z_{1}$, are shown in Fig. 2.

The Ramberg-Osgood stress-strain relation which is used to model the material non-linearity is written as

$$
\varepsilon=\frac{\sigma_{i}}{E_{i}}+\left(\frac{\sigma_{i}}{H_{i}}\right)^{\frac{1}{m_{i}}}
$$

where $\varepsilon$ is the distribution of the lengthwise strains in the cross-section of the left-hand crack arm, $\sigma_{i}$ is the distribution of the normal stresses in the cross-section of the $i$-th layer, $E_{i}$ is the modulus of elasticity in the same layer, $H_{i}$ and $m_{i}$ are 
material properties which describe the hardening behavior of the material (actually, the second term of the right-hand side of (6) models the material non-linearity).

Each layer exhibits smooth material inhomogeneity along the width and length of the layer. Thus, it is assumed that the modulus of elasticity in the $i$-th layer varies continuously along the width according to following cosine law:

$$
E_{i}=E_{d_{i}}+E_{f_{i}} \cos \left(\frac{\pi}{2} \frac{y_{1}-y_{1 i}}{y_{1 i+1}-y_{1 i}}\right)
$$

where

$$
y_{1 i} \leq y_{1} \leq y_{1 i+1} .
$$

In (7), $E_{d_{i}}$ is the value of the modulus of elasticity at the right-hand lateral surface of the layer, $E_{f_{i}}$ is a material property which governs the material gradient along the width. Apparently, the value of the modulus of elasticity at the left-hand lateral surface of the layer is $E_{d_{i}}+E_{f_{i}}$. The continuous variation of $E_{d_{i}}$ in the length direction of the $i$-th layer is written as

$$
E_{d_{i}}=E_{g_{i}}+E_{r_{i}} \cos \left(\frac{\pi}{2} \frac{l_{1}+l_{2}-x_{3}}{l_{1}+l_{2}}\right)
$$

where

$$
0 \leq x_{3} \leq 2\left(l_{1}+l_{2}\right)
$$

The $x_{3}$-axis is shown in Fig. 1. In (9), $E_{g_{i}}$ is the value of $E_{d_{i}}$ at the two end sections, $x_{3}=0$ and $x_{3}=2\left(l_{1}+l_{2}\right)$, of the beam, $E_{r_{i}}$ is a material property which governs the material gradient in the length direction. It is obvious that the value of $E_{d_{i}}$ in the mid-span is $E_{g_{i}}+E_{r_{i}}$.

For the Ramberg-Osgood stress-strain relation, $u_{0 L_{i}}^{*}$ which is needed in order to calculate $U_{L}^{*}$ by (5) can be written as [17, 18]

$$
u_{0 L_{i}}^{*}=\frac{\sigma_{i}^{2}}{2 E_{i}}+\frac{m_{i} \sigma_{i}^{\frac{1+m_{i}}{m_{i}}}}{\left(1+m_{i}\right) H_{i}^{\frac{1}{m_{i}}}}
$$

By substituting of (7) in (11), one arrives at

$$
u_{0 L_{i}}^{*}=\frac{\sigma_{i}^{2}}{2\left[E_{d_{i}}+E_{f_{i}} \cos \left(\frac{\pi}{2} \frac{y_{1}-y_{1 i}}{y_{1 i+1}-y_{1 i}}\right)\right]}+\frac{m_{i} \sigma_{i}^{\frac{1+m_{i}}{m_{i}}}}{\left(1+m_{i}\right) H_{i}^{\frac{1}{m_{i}}}}
$$

In order to perform the integration in (5), $E_{d_{i}}$ in (12) should be expressed as a function of $x_{1}$. For this purpose, (9) is rewritten as

$$
E_{d_{i}}=E_{g_{i}}+E_{r_{i}} \cos \left(\frac{\pi}{2} \frac{\left(-x_{1}\right)}{l_{1}+l_{2}}\right)
$$


The complementary strain energy cumulated in portion, $l_{1}+l_{2}+a \leq x_{3} \leq l_{1}+2 l_{2}$, of the un-cracked part of the beam is written as

$$
U_{\mathrm{R}}^{*}=\sum_{i=1}^{i=n} \int_{a}^{l_{2}} \int_{y_{2 i}}^{y_{2 i+1}} \int_{-\frac{b}{2}}^{\frac{h}{2}} u_{0 R_{i}}^{*} d x_{1} d y_{2} d \tau_{2}
$$

where $n$ is the number of layers in the un-cracked beam portion, $y_{2 i}$ and $y_{2 i+1}$ are the coordinates, respectively, of the left-hand and right-hand lateral surfaces of the $i$-th layer, $u_{0 R_{i}}^{*}$ is the complementary strain energy density in the same layer, $y_{2}$ and $z_{2}$, are the centroidal axes of the cross-section of the un-cracked portion of the beam.

The complementary strain energy density in the $i$-th layer of the un-cracked beam portion can be obtained by (12). For this purpose, $\sigma_{i}, y_{1}, y_{1 i}$ and $y_{1 i+1}$ have to be replaced, respectively, with $\sigma_{R_{i}}, y_{2}, y_{2 i}$ and $y_{2 i+1}$ where $\sigma_{R_{i}}$ is the distribution of the normal stresses in the cross-section of the $i$-th layer of the un-cracked beam portion.

In order to perform the integration in (5), $\sigma_{i}$ has to be presented as a function of $y_{1}$ and $z_{1}$. It is obvious that $\sigma_{i}$ can not be determined explicitly from Eq. (6). Therefore, $\sigma_{i}$ is expanded in series of Taylor by keeping the first six members

$$
\begin{aligned}
& \sigma_{i}\left(y_{1}, z_{1}\right) \approx \sigma_{i}\left(y_{a i}, 0\right)+\frac{\partial \sigma_{i}\left(y_{a i}, 0\right)}{\partial y_{1}}\left(y_{1}-y_{a i}\right)+\frac{\partial \sigma_{i}\left(y_{a i}, 0\right)}{\partial z_{1}} z_{1}+\frac{\partial^{2} \sigma_{i}\left(y_{a i}, 0\right)}{2 ! \partial y_{1}^{2}}\left(y_{1}-y_{a i}\right)^{2}+ \\
& +\frac{\partial^{2} \sigma_{i}\left(y_{a i}, 0\right)}{\partial y_{1} \partial z_{1}}\left(y_{1}-y_{a i}\right) z_{1}+\frac{\partial^{2} \sigma_{i}\left(y_{a i}, 0\right)}{2 ! \partial z_{1}^{2}} z_{1}^{2}
\end{aligned}
$$

where $y_{a i}=\left(y_{1 i}+y_{1 i+1}\right) / 2, y_{1 i} \leq y_{1} \leq y_{1 i+1}$ and $-b / 2 \leq z_{1} \leq b / 2$ (Fig. 2). Formula (15) is re-written as

$$
\sigma_{i}\left(y_{1}, z_{1}\right) \approx \omega_{1 i}+\omega_{2 i}\left(y_{1}-y_{a i}\right)+\omega_{3 i} z_{1}+\omega_{4 i}\left(y_{1}-y_{a i}\right)^{2}+\omega_{5 i}\left(y_{1}-y_{a i}\right) z_{1}+\omega_{6 i} z_{1}^{2}
$$

where the coefficients, $\omega_{1 i}, \omega_{2 i}, \omega_{3 i}, \omega_{4 i}, \omega_{5 i}$ and $\omega_{6 i}$, are determined in the following manner. First, the distribution of lengthwise strains in the cross-section of the left-hand crack arm is written as

$$
\varepsilon=\kappa_{1} z_{1}
$$

where $\kappa_{1}$ is the curvature the left-hand crack arm. It should be noted that formula (17) follows from the fact that validity of the Bernoulli's hypothesis for plane sections is assumed in the present paper since the span to height ratio of the beam under consideration is large. Concerning the application of the Bernoulli's hypothesis for plane sections, it should also be mentioned that since the beam portion, $B_{2} B_{4}$, in which the delamination crack is located, is loaded in pure bending (Fig. 1), the only non-zero strains are the lengthwise strains. Thus, according to the small strains compatibility equations, the lengthwise strains are distributed linearly along the cross-section height.

By substituting of (7), (16) and (17) in (6), one arrives at

$$
\begin{aligned}
& \kappa_{1} z_{1}=\frac{\omega_{1 i}+\omega_{2 i}\left(y_{1}-y_{a i}\right)+\omega_{3 i} z_{1}+\omega_{4 i}\left(y_{1}-y_{a i}\right)^{2}+\omega_{5 i}\left(y_{1}-y_{a i}\right) z_{1}+\omega_{6 i} z_{1}^{2}}{E_{d_{i}}+E_{f_{i}} \cos \left(\frac{\pi}{2} \frac{y_{1}-y_{1 i}}{y_{1 i+1}-y_{1 i}}\right)}+ \\
& +\frac{\left[\omega_{1 i}+\omega_{2 i}\left(y_{1}-y_{a i}\right)+\omega_{3 i} z_{1}+\omega_{4 i}\left(y_{1}-y_{a i}\right)^{2}+\omega_{5 i}\left(y_{1}-y_{a i}\right) z_{1}+\omega_{6 i} z_{1}^{2}\right]^{\frac{1}{m_{i}}}}{H_{i}^{\frac{1}{m_{i}}}}
\end{aligned}
$$


By substituting of $y_{1}=y_{a i}$ and $z_{1}=0$ in (18), one obtains

$$
\omega_{1 i}+\left[\lambda_{i}+v_{i} \cos \left(y_{a i} \delta_{i}-\phi_{i}\right)\right] \omega_{1 i}^{\frac{1}{m_{i}}}=0
$$

where

$$
\begin{aligned}
& \lambda_{i}=\frac{E_{d_{i}}}{\frac{1}{H_{i}^{m_{i}}}} \\
& v_{i}=\frac{E_{f_{i}}^{\frac{1}{m_{i}}}}{H_{i}^{m_{i}}} \\
& \delta_{i}=\frac{\pi}{2\left(y_{1 i+1}-y_{1 i}\right)} \\
& \phi_{i}=\frac{\pi y_{1 i}}{2\left(y_{1 i+1}-y_{1 i}\right)}
\end{aligned}
$$

Further, by substituting of $y_{1}=y_{a i}$ and $z_{1}=0$ in the first derivative of (18) with respect to $y_{1}$, one arrives at

$$
\omega_{2 i}-v_{i} \delta_{i} \sin \left(y_{a i} \delta_{i}-\phi_{i}\right) \omega_{1 i}^{\frac{1}{m_{i}}}+\left[\lambda_{i}+v_{i} \cos \left(y_{a i} \delta_{i}-\phi_{i}\right)\right] \frac{1}{m_{i}} \omega_{1 i}^{\frac{1-m_{i}}{m_{i}}} \omega_{2 i}=0
$$

Similarly, by substituting of $y_{1}=y_{a i}$ and $z_{1}=0$ in the first derivative of (18) with respect to $z_{1}$, one obtains

$$
\kappa_{1} E_{d i}+\kappa_{1} E_{f_{i}} \cos \left(y_{a i} \delta_{i}-\phi_{i}\right)=\omega_{3 i}+\left[\lambda_{i}+v_{i} \cos \left(y_{a i} \delta_{i}-\phi_{i}\right)\right] \frac{1}{m_{i}} \omega_{1 i}^{\frac{1-m_{i}}{m_{i}}} \omega_{3 i}
$$

Further, by substituting of $y_{1}=y_{a i}$ and $z_{1}=0$ in the second derivatives of (18) with respect to $y_{1}, y_{1}$ and $z_{1}$, and $z_{1}$, one arrives at

$$
\begin{aligned}
& 2 \omega_{4 i}-v_{i} \delta_{i}^{2} \cos \left(y_{a i} \delta_{i}-\phi_{i}\right) \omega_{1 i}^{\frac{1}{m_{i}}}-v_{i} \delta_{i} \sin \left(y_{a i} \delta_{i}-\phi_{i}\right) \frac{1}{m_{i}} \omega_{1 i}^{\frac{1-m_{i}}{m_{i}}} \omega_{2 i}+\left\{-v_{i} \delta_{i} \sin \left(y_{a i} \delta_{i}-\phi_{i}\right) \frac{1}{m_{i}} \omega_{1 i}^{\frac{1-m_{i}}{m_{i}}}+\right. \\
& \left.+\left[\lambda_{i}+v_{i} \cos \left(y_{a i} \delta_{i}-\phi_{i}\right)\right] \frac{1-m_{i}}{m_{i}^{2}} \omega_{1 i}^{\frac{1-2 m_{i}}{m_{i}}} \omega_{2 i}\right\} \omega_{2 i}+2\left[\lambda_{i}+v_{i} \cos \left(y_{a i} \delta_{i}-\phi_{i}\right)\right] \frac{1}{m_{i}} \omega_{1 i}^{\frac{1-m_{i}}{m_{i}}} \omega_{4 i}=0 \\
& -\kappa_{1} E_{f_{i}} \delta_{i} \sin \left(y_{a i} \delta_{i}-\phi_{i}\right)=\omega_{5 i}-v_{i} \delta_{i} \sin \left(y_{a i} \delta_{i}-\phi_{i}\right) \frac{1}{m_{i}} \omega_{1 i}^{\frac{1-m_{i}}{m_{i}}} \omega_{3 i}+ \\
& {\left[\lambda_{i}+v_{i} \cos \left(y_{a i} \delta_{i}-\phi_{i}\right)\right]\left(\frac{1-m_{i}}{m_{i}} \omega_{1 i}^{\frac{1-2 m_{i}}{m_{i}}} \omega_{3 i} \omega_{2 i}+\frac{1}{m_{i}} \omega_{1 i}^{\frac{1-m_{i}}{m_{i}}} \omega_{5 i}\right),}
\end{aligned}
$$




$$
2 \omega_{6 i}+\left[\lambda_{i}+v_{i} \cos \left(y_{a i} \delta_{i}-\phi_{i}\right)\right]\left(\frac{1-m_{i}}{m_{i}^{2}} \omega_{1 i}^{\frac{1-2 m_{i}}{m_{i}}} \omega_{3 i}^{2}+\frac{2}{m_{i}} \omega_{1 i}^{\frac{1-m_{i}}{m_{i}}} \omega_{6 i}\right)=0
$$

Eqns. (19), (24) - (28) can be written for each layer of the left-hand crack arm. In this way, $6 n_{L}$ equations with $6 n_{L}+1$ unknowns, $\omega_{1 i}, \omega_{2 i}, \omega_{3 i}, \omega_{4 i}, \omega_{5 i}, \omega_{6 i}$ and $\kappa_{1}$, where $i=1,2, \ldots, n_{L}$, can be constructed. Another equation can be written by considering the equilibrium of the elementary forces in the left-hand crack arm cross-section

$$
M=\sum_{i=1}^{i=n_{L}} \int_{y_{1 i}}^{y_{1 i+1}} \int_{-\frac{h}{2}}^{\frac{h}{2}} \sigma_{i} z_{1} d y_{1} d z_{1}
$$

where $M$ is the bending moment in the left-hand crack arm. It is obvious that (Fig. 1)

$$
M=F l_{1}
$$

By substituting of (16) in (29), one derives

$$
M=\sum_{i=1}^{i=n_{L}}\left[\omega_{3 i}\left(y_{1 i+1}-y_{1 i}\right) \frac{b^{3}}{12}+\omega_{5 i}\left[\left(y_{1 i+1}-y_{a i}\right)^{2}-\left(y_{1 i}-y_{a i}\right)^{2}\right] \frac{b^{3}}{24}\right]
$$

Eqns. (19), (24) - (28) and (31) should be solved with respect to $\omega_{1 i}, \omega_{2 i}, \omega_{3 i}, \omega_{4 i}, \omega_{5 i}, \omega_{6 i}$ and $\kappa_{1}$ by using the MatLab computer program.

Formula (16) is applied also to present $\sigma_{R_{i}}$ as a function of $y_{2}$ and $z_{2}$. For this purpose, $\omega_{1 i}, \omega_{2 i}, \omega_{3 i}, \omega_{4 i}, \omega_{5 i}, \omega_{6 i}$, $y_{1}$ and $z_{1}$ are replaced with $\omega_{1 R i}, \omega_{2 R i}, \omega_{3 R i}, \omega_{4 R i}, \omega_{5 R i}, \omega_{6 R i}, y_{2}$ and $z_{2}$, respectively. It should be noted that Eqs. (19), (24) - (28) and (31) can be used also to determine $\omega_{1 R i}, \omega_{2 R i}, \omega_{3 R i}, \omega_{4 R i}, \omega_{5 R i}, \omega_{6 R i}$ and $\kappa_{2}$ where $\kappa_{2}$ is the curvature of the portion, $l_{1}+l_{2}+a \leq x_{3} \leq l_{1}+2 l_{2}$, of the un-cracked part of the beam. For this purpose, $n_{L}, y_{1 i}, y_{1 i+1}, \omega_{1 i}, \omega_{2 i}, \omega_{3 i}$ , $\omega_{4 i}, \omega_{5 i}, \omega_{6 i}$ and $\kappa_{1}$ are replaced, respectively, with $n, y_{2 i}, y_{2 i+1}, \omega_{1 R i}, \omega_{2 R i}, \omega_{3 R i}, \omega_{4 R i}, \omega_{5 R i}, \omega_{6 R i}$ and $\kappa_{2}$ in (19), (22) $-(28)$ and (31).

By substituting of (4), (5) and (14) in (3), one arrives at

$$
G=2\left(\frac{1}{b} \sum_{i=1}^{i=n_{L}} \int_{y_{1 i}}^{y_{1 i+1}} \int_{-\frac{h}{2}}^{\frac{b}{2}} u_{0 L_{i}}^{*} d y_{1} d x_{1}-\frac{1}{b} \sum_{i=1}^{i=n} \int_{y_{2 i}}^{y_{2 i+1}} \int_{-\frac{h}{2}}^{\frac{b}{2}} u_{0 R_{i}}^{*} d y_{2} d z_{2}\right)
$$

where $u_{0 L_{i}}^{*}$ and $u_{0 R_{i}}^{*}$ are obtained by (12), (13), (16), (19), (20), (23), (25)-(28) and (31) at $x_{1}=a$. It should be noted that the term in the brackets in (32) is doubled in view of the symmetry (Fig. 1). The integration in (32) should be carried-out by using the MatLab computer program.

The delamination fracture behavior is analyzed also by applying the $J$-integral approach [19] in order to verify the solution to the strain energy release rate (32). The integration of the $J$-integral is performed along the integration contour, $\Gamma$, showed by a dashed line in Fig. 1. Since the right-hand crack arm is free of stresses, the solution of the J-integral is written as

$$
J=2\left(J_{\Gamma_{1}}+J_{\Gamma_{2}}\right)
$$

where $J_{\Gamma_{1}}$ and $J_{\Gamma 2}$ are the values of the $J$-integral, respectively, in segments, $\Gamma_{1}$ and $\Gamma_{2}$, of the integration contour (segments, $\Gamma_{1}$ and $\Gamma_{2}$, coincide with the cross-section of the left-hand crack arm and un-cracked beam portion, respectively). The term in brackets in (33) is doubled because of the symmetry. 
The $J$-integral in segment, $\Gamma_{1}$, is written as

$$
J_{\Gamma_{1}}=\sum_{i=1}^{i=n_{L}} \int_{y_{1 i}}^{y_{1 i+1}}\left[u_{0 L_{i}} \cos \alpha-\left(p_{x i} \frac{\partial u}{\partial x}+p_{y i} \frac{\partial v}{\partial x}\right)\right] d s
$$

where $u_{0 L_{i}}$ is the strain energy density in the $i$-th layer of the left-hand crack arm, $\alpha$ is the angle between the outwards normal vector to the contour of integration and the crack direction, $p_{x i}$ and $p_{y i}$ are the components of stress vector in the $i$-th layer of the left-hand crack arm, $u$ and $v$ are the components of displacement vector with respect to the crack tip coordinate system $x y$ ( $x$ is directed along the delamination crack), $d s$ is a differential element along the contour of integration. The strain energy density in the $i$-th layer of the left-hand crack arm is obtained by applying the following formula [17, 18]:

$$
u_{0 L_{i}}=\frac{\sigma_{i}^{2}}{2 E_{i}}+\frac{\sigma_{i}^{\frac{1+m_{i}}{m_{i}}}}{\left(1+m_{i}\right) H_{i}^{\frac{1}{m_{i}}}}
$$

By substituting of (7) in (35), one obtains

$$
u_{0 L_{i}}=\frac{\sigma_{i}^{2}}{2\left[E_{d_{i}}+E_{f_{i}} \cos \left(\frac{\pi}{2} \frac{y_{1}-y_{1 i}}{y_{1 i+1}-y_{1 i}}\right)\right]}+\frac{\sigma_{i}^{\frac{1+m_{i}}{m_{i}}}}{\left(1+m_{i}\right) H_{i}^{\frac{1}{m_{i}}}} .
$$

The other components of the $J$-integral in segment, $\Gamma_{1}$, are written as

$$
\begin{aligned}
& p_{x i}=-\sigma_{i} \\
& p_{y i}=0 \\
& d s=d y_{1} \\
& \cos \alpha=-1
\end{aligned}
$$

It should be noted that formula (16) is used to obtain the stress, $\sigma_{i}$, in (37). The partial derivative, $\partial u / \partial x$, that is involved in (34) is expressed as

$$
\frac{\partial u}{\partial x}=\varepsilon=\kappa_{1} z_{1}
$$

The $J$-integral is segment, $\Gamma_{2}$, is written as

$$
J_{\Gamma_{2}}=\sum_{i=1}^{i=n} \int_{y_{2 i}}^{y_{2 i+1}}\left[u_{0 R_{i}} \cos \alpha_{\mathrm{R}}-\left(p_{x \mathrm{R} i} \frac{\partial u}{\partial x_{\mathrm{R}}}+p_{y \mathrm{R} i} \frac{\partial v}{\partial x_{\mathrm{R}}}\right)\right] d s_{\mathrm{R}}
$$

where the strain energy density, $u_{0 R_{i}}$, in the $i$-th layer of the un-cracked beam portion is obtained by formula (36). For this purpose, $\sigma_{i}, y_{1}, y_{1 i}$ and $y_{1 i+1}$ are replaced, respectively, with $\sigma_{R_{i}}, y_{2}, y_{2 i}$ and $y_{2 i+1}$. The other components of $J_{\Gamma_{2}}$ are written as 


$$
\begin{aligned}
& p_{x \mathrm{R} i}=\sigma_{\mathrm{R}_{i}} \\
& p_{y \mathrm{R} i}=0 \\
& d s_{\mathrm{R}}=-d y_{2} \\
& \cos \alpha_{\mathrm{R}}=1 \\
& \frac{\partial u}{\partial x_{\mathrm{R}}}=\kappa_{2} z_{2}
\end{aligned}
$$

Formula (16) is applied to calculate the stress, $\sigma_{R_{i}}$, in (43). For this purpose, $\omega_{1 i}, \omega_{2 i}, \omega_{3 i}, \omega_{4 i}, \omega_{5 i}, \omega_{6 i}, y_{1}$ and $z_{1}$ are replaced with $\omega_{1 R i}, \omega_{2 R i}, \omega_{3 R i}, \omega_{4 R i}, \omega_{5 R i}, \omega_{6 R i}, y_{2}$ and $z_{2}$, respectively.

The average value of the $J$-integral along the delamination crack front is written as

$$
J_{a v}=\frac{1}{b} \int_{-\frac{b}{2}}^{\frac{b}{2}} J d z_{1}
$$

By substituting of (33), (34) and (42) in (48), one arrives at

$$
\begin{aligned}
& J_{a v}=\frac{2}{b}\left\{\sum_{i=1}^{i=n_{\mathrm{L}}} \int_{-\frac{h}{2}}^{\frac{h}{2}} \int_{y_{1 i}}^{y_{1 i+1}}\left[u_{0 L_{i}} \cos \alpha-\left(p_{x i} \frac{\partial u}{\partial x}+p_{y i} \frac{\partial v}{\partial x}\right)\right] d s+\right. \\
& \left.+\sum_{i=1}^{i=n} \int_{-\frac{h}{2}}^{\frac{b}{2}} \int_{y_{2 i}}^{y_{2 i+1}}\left[u_{0 R_{i}} \cos \alpha_{\mathrm{R}}-\left(p_{x \mathrm{R} i} \frac{\partial u}{\partial x_{\mathrm{R}}}+p_{y \mathrm{R} i} \frac{\partial v}{\partial x_{\mathrm{R}}}\right)\right] d s_{\mathrm{R}}\right\}
\end{aligned}
$$

where $u_{0 L_{i}}, u_{0 R_{i}}, p_{x i}$ and $p_{x R i}$ are obtained by (16), (19), (20), (23), (25) - (28), (31), (36), (37) and (43) at $x_{1}=a$. The integration in (49) should be carried-out by the MatLab computer program. The $J$-integral value obtained by (49) matches exactly the strain energy release rate determined by (32). This fact is a verification of the delamination fracture analysis developed in the present paper. It should be mentioned that the delamination fracture is analyzed also by keeping more than six members in the in series of Taylor (15). The results obtained are very close to these derived by keeping six members (the difference is less than $2 \%$ ).

\section{PARAMETRIC STUDY}

$\mathrm{E}$ ffects of material inhomogeneity in width and length directions, crack location along the beam width, non-linear mechanical behavior of the material and crack length on the delamination fracture in the multilayered four-point bending beam are investigated by applying the solution to the strain energy release rate (32). The results obtained are presented in non-dimensional form by using the formula $G_{N}=G /\left(E_{g_{1}} b\right)$. Two three-layered four-point bending beam configurations are considered in order to evaluate the influence of the delamination crack location along the beam width on the fracture behavior (Fig. 3).

A beam configuration with a delamination crack located between layers 2 and 3 is shown in Fig. 3a. A beam with a delamination crack between layers 1 and 2 is also considered (Fig. 3b). The width of each layer is $t$. It is assumed that $t=0.004 \mathrm{~m}, h=0.016 \mathrm{~m}, l_{1}=0.100 \mathrm{~m}, l_{2}=0.150 \mathrm{~m}$ and $F=15 \mathrm{~N}$. 
The strain energy release rate in non-dimensional form is presented as a function of $H_{2} / H_{1}$ ratio in Fig. 4 for the two beam configurations shown in Fig. 3 at $a / l_{2}=0.75, E_{g_{2}} / E_{g_{1}}=0.6, E_{g_{3}} / E_{g_{1}}=0.5, E_{r_{1}} / E_{g_{1}}=0.4, E_{r_{2}} / E_{r_{1}}=0.6$, $E_{r_{3}} / E_{r_{1}}=0.8, E_{f_{1}} / E_{g_{1}}=0.6, E_{f_{2}} / E_{f_{1}}=0.7, E_{f_{3}} / E_{f_{1}}=1.1, H_{1} / E_{g_{1}}=0.6, H_{3} / H_{1}=0.7$ and $m_{1}=m_{2}=m_{3}=0.7$.

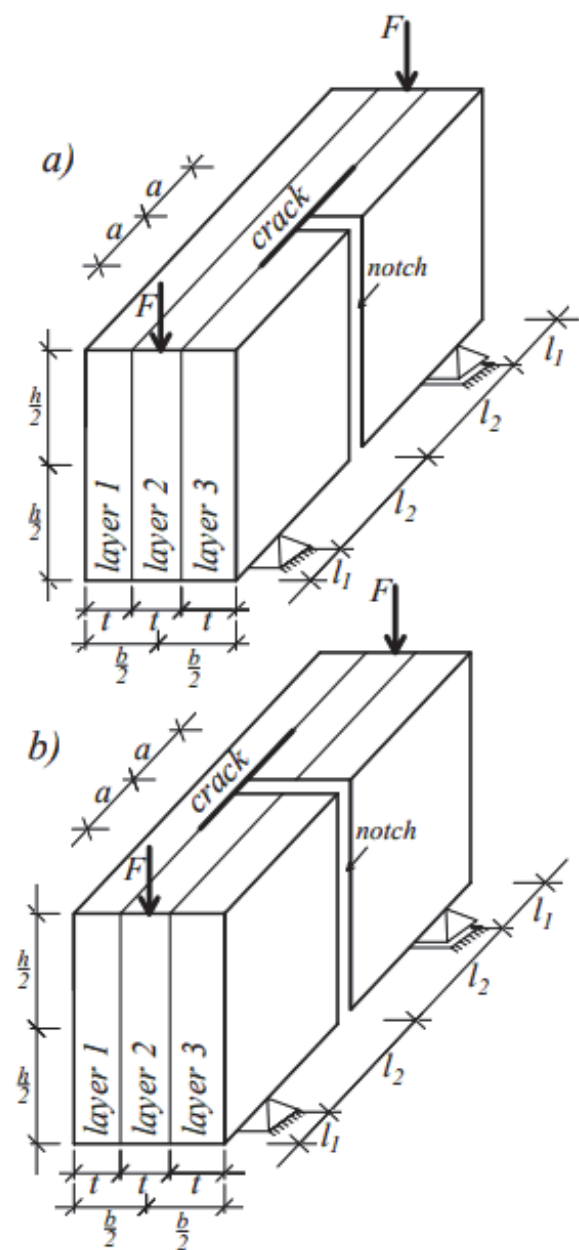

Figure 3: Two three-layered four-point bending beam configurations with delamination crack located between (a) layers 2 and 3 and (b) layers 1 and 2 .

The curves in Fig. 4 indicate that the strain energy release rate decreases with increasing of $H_{2} / H_{1}$ ratio. Besides, it can be observed in Fig. 4 that the strain energy release rate is higher when the delamination crack is located between layers 1 and 2. This behavior is due to the fact that when the delamination crack is located between layers 1 and 2 , the stiffness of lefthand crack arm is lower.

The influence of the $E_{g_{2}} / E_{g_{1}}$ ratio on the delamination fracture is investigated too. For this purpose, the strain energy release rate in non-dimensional form is presented as a function $E_{g_{2}} / E_{g_{1}}$ ratio in Fig. 5. The three-layered four-point bending beam configuration with a delamination crack located between layers 1 and 2 is analyzed (Fig. 3b). It can be observed in Fig. 5 that the strain energy release rate decreases with increasing of $E_{g_{2}} / E_{g_{1}}$ ratio.

The effect of the non-linear mechanical behavior of the material is elucidated also. For this purpose, the strain energy release rate obtained assuming linear-elastic behavior of the inhomogeneous material in each layer is presented also in Fig. 5 for comparison with the non-linear solution.

The linear-elastic solution is derived by substituting of $H_{i} \rightarrow \infty$ in formulae (12), (19), (20) and (32) since at $H_{i} \rightarrow \infty$ the Ramberg-Osgood stress-strain relation (6) transforms in the Hooke's law. One can observe that the non-linear behavior of the material leads to increase of the strain energy release rate (Fig. 5). 


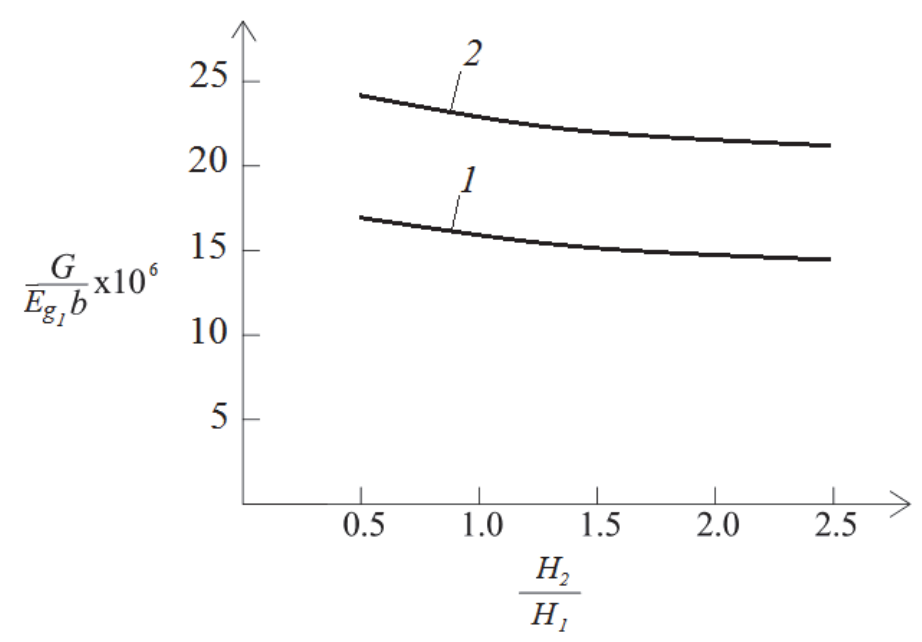

Figure 4: The strain energy release rate in non-dimensional form presented as a function of $H_{2} / H_{1}$ ratio (curve 1 - for the beam configuration with delamination located between layers 2 and 3 (refer to Fig. 3a), curve 2 - for the beam configuration with delamination located between layers 1 and 2 (refer to Fig. 3b)).

The effect of material inhomogeneity along the width of layer 1 on the delamination fracture is analyzed (the beam configuration shown in Fig. $3 \mathrm{~b}$ is considered). For this purpose, the strain energy release rate in non-dimensional form is presented as a function of $E_{f_{1}} / E_{d_{1}}$ ratio in Fig. 6 at two $H_{1} / E_{d_{1}}$ ratios. It can be observed in Fig. 6 that the strain energy release rate decreases with increasing of $E_{f_{1}} / E_{d_{1}}$ and $H_{1} / E_{d_{1}}$ ratios.

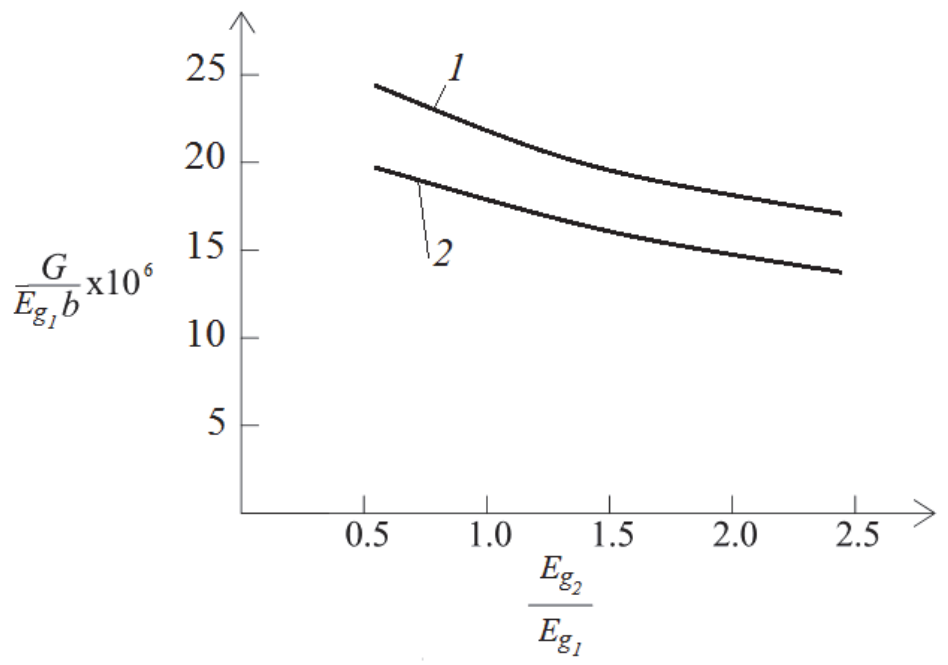

Figure 5: The strain energy release rate in non-dimensional form presented as a function of $E_{g_{2}} / E_{g_{1}}$ ratio (curve 1 - at non-linear mechanical behavior of the material, curve 2 - at linear-elastic behavior).

The influences of the material inhomogeneity in the beam length direction and the delamination crack length on the fracture behavior are studied. For this purpose, the strain energy release rate in non-dimensional form is presented as a function of $E_{r_{1}} / E_{g_{1}}$ in Fig. 7 at three $a / l_{2}$ ratios for the three-layered beam configuration shown in Fig. $3 \mathrm{~b}$.

The curves in Fig. 7 indicate that the strain energy release rate decreases with increasing of $E_{r_{1}} / E_{g_{1}}$ ratio.

One can observe also in Fig. 7 that the strain energy release rate increases with increasing of $a / l_{2}$ ratio (this founding is attributed to the fact that the modulus of elasticity in the beam cross-section in which the delamination crack front is located decreases with increasing of the crack length). 


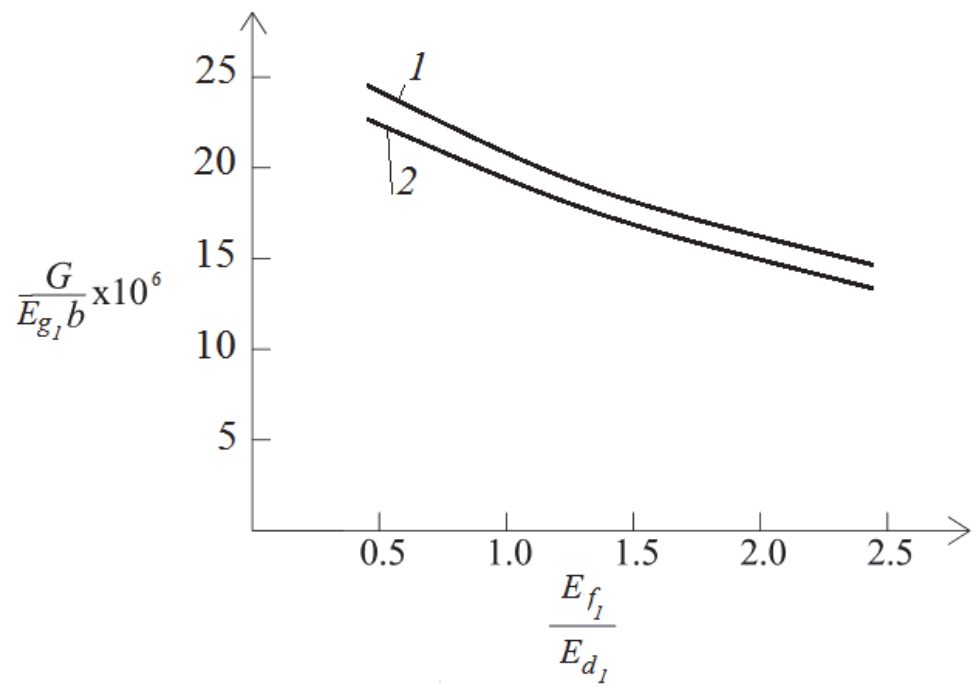

Figure 6: The strain energy release rate in non-dimensional form presented as a function of $E_{f_{1}} / E_{d_{1}}$ ratio (curve $1-$ at $H_{1} / E_{d_{1}}=0.5$ curve 2 - at $\left.H_{1} / E_{d_{1}}=4\right)$.

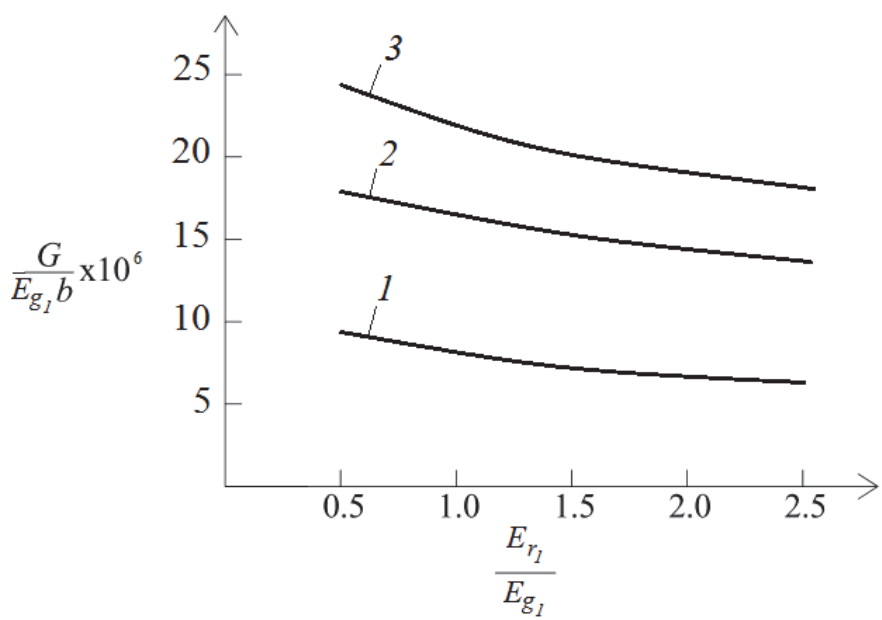

Figure 7: The strain energy release rate in non-dimensional form presented as a function of $E_{r_{1}} / E_{g_{1}}$ ratio (curve $1-$ at $a / l_{2}=0.25$, curve 2 - at $a / l_{2}=0.5$ and curve 3 - at $\left.a / l_{2}=0.75\right)$.

\section{CONCLUSIONS}

A solution to the strain energy release rate for a delamination crack in multilayered four-point bending beam configurations is derived assuming that each layer exhibits smooth material inhomogeneity in both width and length directions. The solution is intended for brittle materials. Cosine laws are adopted in order to describe the continuous variation of the modulus of elasticity along the width and length of layers. The beam is made by adhesively bonded lengthwise vertical layers which have individual widths and material properties. The number of layers is arbitrary. The material in each layer exhibits non-linear mechanical behavior that is modeled by applying the Ramberg-Osgood equation. The solution derived holds for a delamination crack that is located arbitrary between layers. The delamination fracture is analyzed also by applying the $J$-integral approach in order to verify the solution to the strain energy release rate. It should be mentioned that the delamination fracture analysis developed in the present paper is valid for non-linear elastic behavior of the material. However, the analysis can also be applied for elastic-plastic behavior if the multilayered beam 
under consideration undergoes active deformation, i.e. if the external loading increases only [20,21]. The influence of material inhomogeneity along the width and length of layers is elucidated. It is found that the strain energy release rate decreases with increasing of $E_{f_{1}} / E_{d_{1}}$ and $E_{r_{1}} / E_{g_{1}}$ ratios. The effect of crack location along the beam width is evaluated too. The analysis reveals that the strain energy release rate decreases with increasing of the width of the left-hand crack arm. Concerning the influence of the delamination crack length on the fracture behavior, it is found that the strain energy release rate increases with increasing of the crack length. The approach developed in the present paper can be useful for evaluation of the effects of material inhomogeneity and non-linear mechanical behavior of the material on delamination fracture in design of multilayered beam structures.

\section{REFERENCES}

[1] Banea, M.D., da Silva, L.F.M. (2016). Adhesively bonded joints in composite materials: An overview, Proceedings of the Institution of Mechanical Engineers, Part L: Journal of Materials: Design and Applications, 223, pp. 1-18.

[2] Wicaksono, S., Chai, G.B. (2012). A review of advances in fatigue and life prediction of fiber-reinforced composites, Proceedings of the Institution of Mechanical Engineers, Part L: Journal of Materials: Design and Applications, 227, pp. 179-195.

[3] Long, L., Huang, Y., Zhang, J. (2015). Experimental investigation and numerical simulation on continuous wave laser ablation of multilayer carbon fiber composite, Proceedings of the Institution of Mechanical Engineers, Part L: Journal of Materials: Design and Applications, 231, pp. 674-682.

[4] Al-Khanbashi, A., Hamdy, A.E. (2004). Fracture mechanics approach to predict delamination lifetime in Mode II under constant loads, Journal of Adhesion Science and Technology, 18, pp. 227-242.

[5] Sayyad, A.S., Ghugal, Y.M. (2018). Modeling and analysis of functionally graded sandwich beams: A review, Mechanics of Advanced Materials and Structures, 1, pp. 1-20.

[6] Rizov, V.I., (2017). Delamination of Multilayered Functionally Graded Beams with Material Nonlinearity, International Journal of Structural Stability and Dynamics, 18(4), 1850051. DOI: 10.1142/S0219455418500517.

[7] Rizov, V.I., (2017). Non-linear elastic delamination of multilayered functionally graded beam, Multidiscipline Modeling in Materials and Structures, 13, pp. 434-447.

[8] Mortensen, A., Suresh, S. (1995). Functionally graded metals and metal-ceramic composites: Part 1 Processing, International Materials Review, 40, pp. 239-265.

[9] Gasik, M.M. (1995). Functionally graded materials: bulk processing techniques, International Journal of Materials and Product Technology, 39, pp. 20-29.

[10] Neubrand, A., Rödel, J. (1997). Gradient materials: An overview of a novel concept, Zeit f Met, 88, pp. 358-371.

[11] Suresh, S., Mortensen, A. (1998). Fundamentals of functionally graded materials, IOM Communications Ltd, London .

[12] Hirai, T., Chen, L. (1999). Recent and prospective development of functionally graded materials in Japan, Material Science Forum, 308-311, pp. 509-514.

[13] Butcher, R.J., Rousseau, C.E., Tippur, H.V. (1999). A functionally graded particulate composite: Measurements and Failure Analysis, Acta Matererialia, 47, pp. 259-268.

[14] Nemat-Allal, M.M., Ata, M.H., Bayoumi, M.R., Khair-Eldeen, W. (2011). Powder metallurgical fabrication and microstructural investigations of Aluminum/Steel functionally graded material, Materials Sciences and Applications, 2, pp. 1708-1718.

[15] Bohidar, S.K., Sharma, R., Mishra, P.R., (2014). Functionally graded materials: A critical review, International Journal of Research, 1, pp. 289-301.

[16] Rizov, V.I., (2017). Analysis of longitudinal cracked two-dimensional functionally graded beams exhibiting material non-linearity, Frattura ed Integrità Strutturale, 41, pp. 498-510.

[17] Rizov, V.I. (2017). Delamination analysis of a layered elastic-plastic beam, International Journal of Structural Integrity, 4, pp. 516-529.

[18] Rizov, V.I. (2018). Lengthwise fracture analyses of functionally graded beams by the Ramberg-Osgood equation, Engineering Review, 38, pp. 309-320.

[19] Broek, D. (1986). Elementary engineering fracture mechanics, Springer.

[20] Lubliner, J. (2006). Plasticity theory (Revised edition), University of California, Berkeley, CA .

[21] Chakrabarty, J. (2006). Theory of plasticity, Elsevier Butterworth-Heinemann, Oxford. 